\title{
INVESTIGATION OF SELF-DIFFUSION OF TITANIUM BY FLUCTUATION AUTOCORRELATION METHOD
}

\author{
A.M. Dąbrowski, T. Biernat, J. Bęben and W. Gubernator \\ Institute of Experimental Physics, Wrocław University \\ Pl. Maxa Borna 9, 50-204 Wrocław, Poland
}

(Received December 17, 1996)

\begin{abstract}
Diffusion of titanium was examined in the ficld emission microscope using the fluctuation autocorrelation method. A thick titanium layer (about three monolayers) deposited on a tungsten tip was used. A temperature range of $1260-1410 \mathrm{~K}$ was found where diffusion can be described in a hydrodynamic approximation. In this temperature range the diffusion coefficient seems to comply with Arrhenius rule for the activation energy of $1.31 \mathrm{eV}$ and $D_{0}=3 \times 10^{-7} \mathrm{~cm}^{2} / \mathrm{s}$.

PACS numbers: $79.70 .+q, 68.90 .+\mathrm{g}$
\end{abstract}

\section{Introduction}

Surface phenomena occurring on or with titanium as a substrate or adsorbate, including electron emission and surface diffusion processes, have been studied for many years [1-4] due to a great role played by the metal in engineering and ultrahigh vacuum technology. The interest in this metal, like in some others, has still increased for the possibility to employ it in power engineering as a high capacity material for non-pressure storage of ecologically clean fuel.

In this work, we examined surface self-diffusion as a function of temperature, of a clean titanium deposited onto a field emitter of tungsten, by using the field cmission (FE) fluctuation method. The temperature behavior of clean titanium in ultrahigh vacuum is of primary importance in studies of its interactions with gases. The FE fluctuation method [5-7] of investigation of diffusion and surface interactions is applied to many adsorption systems of both gases on metals (e.g. $\mathrm{CO}$ and oxygen [8]) and metals on metals $(\mathrm{K} / \mathrm{W}, \mathrm{Li} / \mathrm{W}$ [9], $\mathrm{K}$ microcrystals [10], Hf/W [11]). Fluctuations of the adsorbate density, as observed via FE current fluctuations and their autocorrelation function, enable to determine the diffusion and self-diffusion coefficients for chemisorbed layers. Some related surface phenomena were also examined, such as phase transitions and critical fluctuations $[7,9,12]$. 


\section{Experimental}

The measurements were carried out in a sealed-oft, glass field FE microscope equipped with a Faraday collector, a titanium source, Bayard-Alpert gauge and an ion getter pump. Prior to the sealing off, the microscope was thoroughly baked and degassed. The residual gas pressure of the $\mathrm{FE}$ tube was in the $10^{-8} \mathrm{~Pa}$ range. Titanium was evaporated in situ onto the $\mathrm{W}$ tip from a source made of a $\mathrm{Ti}$ block which could be heated by the electron bombardment. The FE image could be observed on a phosphor screen and photographically recorded. The emitter temperature could be changed from $80 \mathrm{~K}$ to high values; it was stabilized by means of a control unit and using the temperature dependence of the resistant of an emitter loop segment which had potential leads.

Prior to the deposition of titanium, the $\mathrm{W}$ tip was cleaned by flashing the emitter over $2300 \mathrm{~K}$. Titanium was evaporated onto the tungsten substrate held at room temperature. After each dose of Ti the emitter was annealed at about $1100 \mathrm{~K}$ to have the titanium spread uniformly over the substrate surface. The changes of the average work function of the system observed during the titanium deposition were determined from the slope of $F-N$ plots.

A diagram of the experimental setup is shown in Fig. 1. The surface mo-

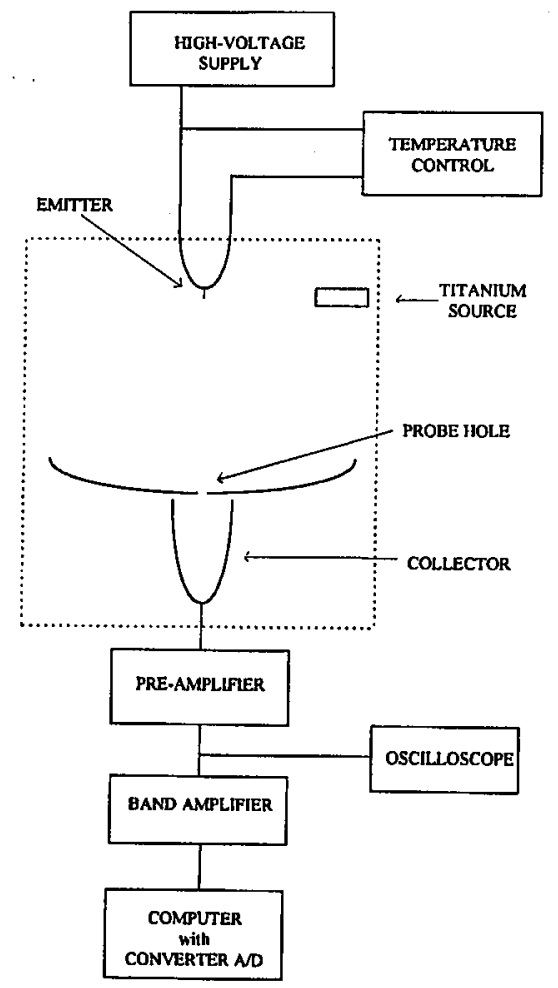

Fig. 1. Experimental setup. 
bility of the obtained two-three monolayers of titanium was investigated from fluctuations of the emission current monitored. The current from the region of the (013) plane of tungsten single crystal was magnetically directed to the collector (an area which corresponds to the probe region of approximately $10^{-12} \mathrm{~cm}^{2}$ ). After amplification of the probe hole current, its ac component was processed in an analog-digital converter PC card. The sampling time was $2 \times 10^{-5} \mathrm{~s}$ and the realization consisted of 400000 samples.

\section{Results and conclusion}

The theoretical autocorrelation functions were calculated from the expression [6]:

$$
R(\tau) / R(0)=1-\exp \left(-2 \alpha^{2}\right)\left[I_{0}\left(2 \alpha^{2}\right)+I_{1}\left(2 \alpha^{2}\right)\right],
$$

where $\alpha^{2}=r_{0}^{2} / 4 D \tau, r_{0}$ is the radius of the probe region, $D$ is diffusion coefficient, $I_{0}$ and $I_{1}$ are the modified Bessel functions. Then, a fitting procedure was applied to compare the theoretical and experimental autocorrelation functions and find the diffusion coefficient.

In order to control the thickness of the titanium adlayers it was necessary first to investigate the behavior of the work function during the course of deposition
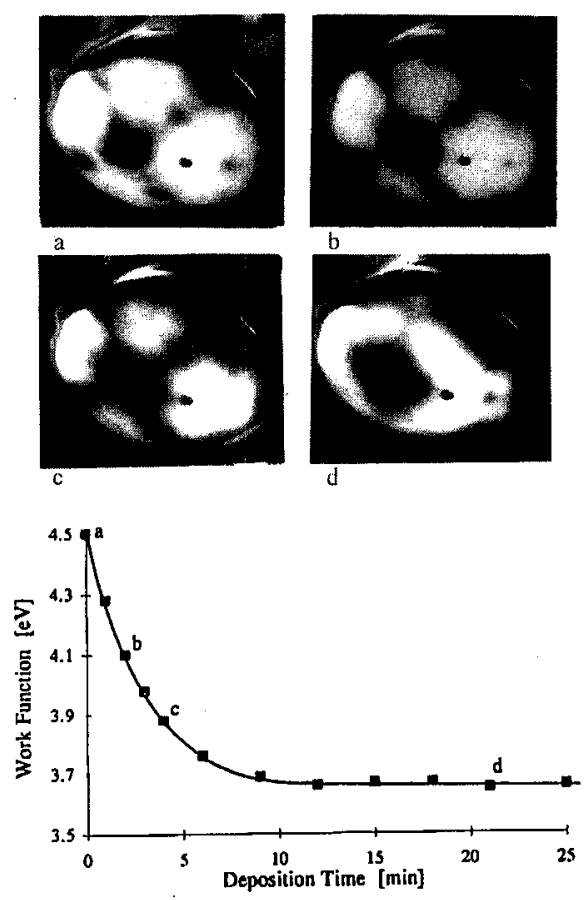

Fig. 2. The typical changes of the average work function versus deposition time of titanium, and examples of the field emission patterns illustrating the adsorption system $\mathrm{Ti} / \mathrm{W}$. 


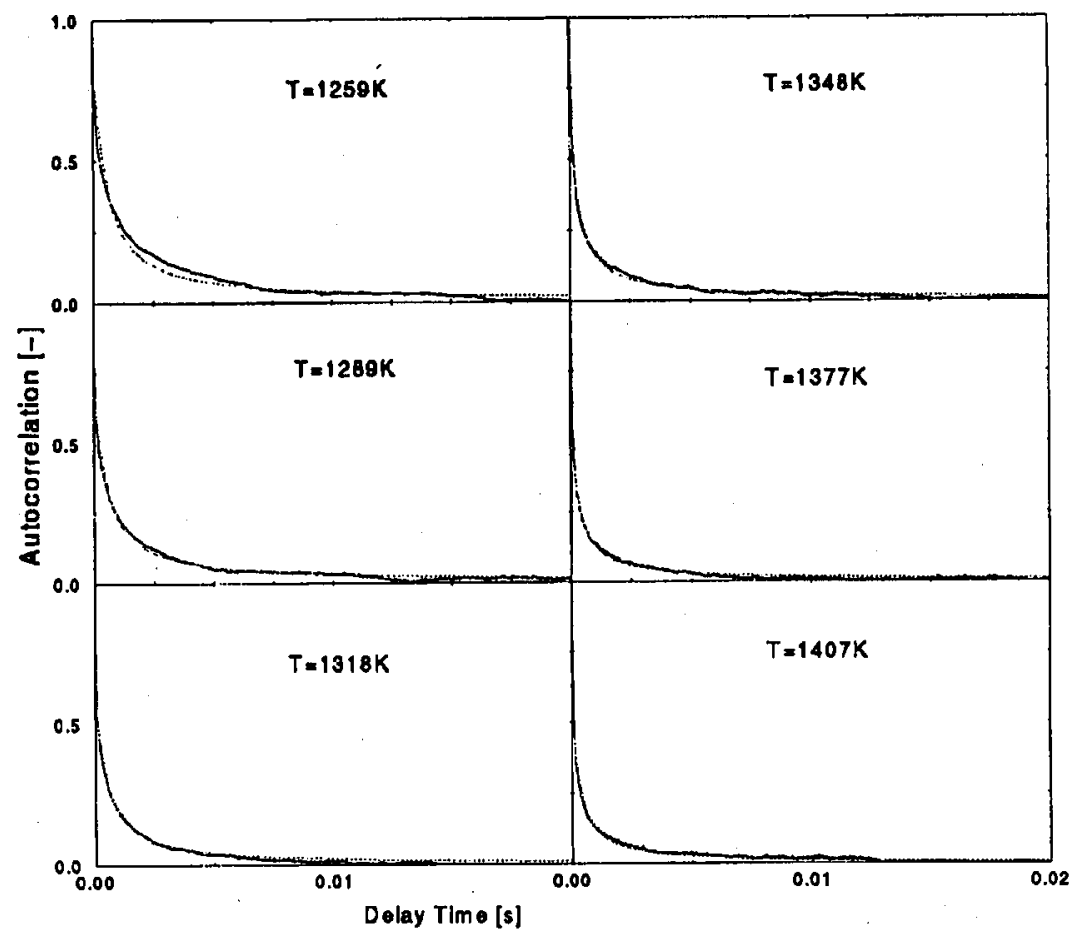

Fig. 3. Examples of the autocorrelation functions at different temperatures. Dashed lines are theoretical autocorrelation functions.

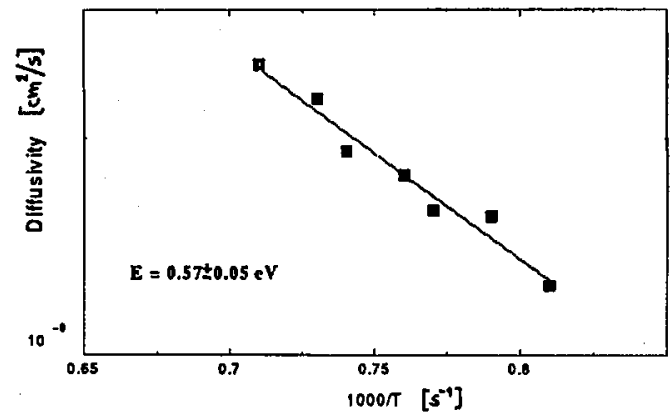

Fig. 4. Temperature dependence of the diffusion coefficients.

of titanium. As it was expected, the average work function measured versus the deposition time (corresponding to coverage) is typical of an electropositive adsorbate. Figure 2 shows such dependence; the selected FE patterns correspond to some characteristic stages of titanium deposition. The initial portion of saturation of the curve, which begins from about 10 minute of the deposition, corresponds to one monolayer of titanium. The results are in general agreement with other, former ones [1-4]. 
The surface self-diffusion of two-three titanium monolayers was investigated in the temperature range $300-1410 \mathrm{~K}$. The measured experimental autocorrelation functions together with the corresponding theoretical curves are shown in Fig. 3 for a temperature range of $1259-1407 \mathrm{~K}$ which appeared to be the range where diffusion can be described in a hydrodynamic approximation using Eq. (1). In this temperature range the diffusion seems to be thermally activated, $D=D_{0} \exp (Q / k T)$, yielding an Arrhenius straight line of a semi-log plot. The Arrhenius-type plot of $\ln D$ vs. reciprocal temperature for the above range of temperature is shown in Fig. 4. The activation energy for self-diffusion of the titanium adlayer was calculated to be $E=1.31 \mathrm{eV}$, and the diffusion coefficient $D_{0}=3 \times 10^{-7} \mathrm{~cm}^{2} / \mathrm{s}$.

\section{Acknowledgments}

The authors would like to thank Professor R. Męclewski for stimulating discussions. We are indebted to S. Surma for his assistance in the measurements of temperature. The work was supported by the Committee for Scientific Research within the framework of the project No. 2 P303120 06p02.

\section{References}

[1] Yu.V. Zubenko, Radiotekhn. Electron. 8, 1239 (1963).

[2] J.R. Anderson, N. Thompson, Surf. Sci. 26, 397 (1971); Sov. Phys.-Solid State 12, 1118 (1970).

[3] G.G. Vladimirov, B.K. Medvedev, I.L. Sokolska, Solid State Phys. 12, 1423 (1970).

[4] Ch.S. Bhatia, M.K. Sinha, Surf. Sci. 43, 369 (1974).

[5] R. Gomer, Surf. Sci. 38, 373 (1973).

[6] M.A. Gesley, L.W. Swanson, Phys. Rev. B 32, 7703 (1985).

[7] G. Mazenko, J.R. Banavar, R. Gomer, Surf. Sci. 107, 459 (1981).

[8] J.R. Chen, R. Gomer, Surf. Sci. 79, 413 (1979); ibid. 81, 589 (1979).

[9] J. Bęben, Solid State Phenom. 12, 17 (1990); A.M. Dąbrowski, Solid State Phenom. 12, 39 (1990); T. Biernat, Solid State Phenom. 12, 61 (1990).

[10] T. Biernat, Ch. Kleint, R. Męclewski, Surf. Sci. 127, 487 (1983).

[11] J. Bęben, W. Gubernator, Surf. Sci. 304, 59 (1994).

[12] B. Bell, R. Gomer, H. Reiss, Surf. Sci. 55, 494 (1976). 